\title{
Novel biomarkers of childhood and adolescent obesity
}

\author{
Licy L. Yanes Cardozo $1,2,3,4,5$. Damian G. Romero ${ }^{1,3,4,5}$
}

Received: 21 February 2021 / Accepted: 28 February 2021 / Published online: 14 April 2021

(c) The Japanese Society of Hypertension 2021

Childhood and adolescent obesity is a worrisome public health concern in the United States and worldwide. The World Health Organization (WHO) estimated that 38.2 million children under the age of 5 years were overweight (OW) or obese (OB) worldwide in 2019 [1]. Moreover, the WHO estimated that over 340 million children and adolescents aged 5-19 were OW or OB in 2016. Once considered a high-income country problem, OW and obesity are now on the rise in low- and middle-income countries, with a particular impact in urban populations [2]. In the USA, the prevalence of obesity among children and adolescents aged 2-19 years was $18.5 \%$ in 2015-2016 [3]. Moreover, the prevalence of obesity among non-Hispanic black $(22.0 \%)$ and Hispanic (25.8\%) children and adolescents was higher than that among both non-Hispanic white $(14.1 \%)$ and non-Hispanic Asian (11.0\%) children and adolescents aged 2-19 years [3]. Furthermore, there was a continuous $\sim 30 \%$ increase in the prevalence of obesity (18.5 vs. $13.9 \%$ ) in children and adolescents aged 2-19 years from 1999-2000 through 2015-2016. Those studies highlight that childhood and adolescent obesity is a global problem with no end in sight in the near future.

Licy L. Yanes Cardozo

lyanes@umc.edu

$\bowtie$ Damian G. Romero dromero@umc.edu

1 Department of Cell and Molecular Biology, University of Mississippi Medical Center, Jackson, MS, USA

2 Department of Medicine, University of Mississippi Medical Center, Jackson, MS, USA

3 Mississippi Center of Excellence in Perinatal Research, University of Mississippi Medical Center, Jackson, MS, USA

4 Women's Health Research Center, University of Mississippi Medical Center, Jackson, MS, USA

5 Cardiovascular-Renal Research Center, University of Mississippi Medical Center, Jackson, MS, USA
The currently ongoing COVID-19 pandemic is likely to worsen the childhood and adolescent obesity crisis. Several studies have reported that during the COVID-19 pandemic lockdown, there was an increase in body mass index (BMI), prevalence of OW/obesity, leisure screen time, and sleeping time and a decrease in physical activity in children and adolescents in multiple countries [4-7]. Moreover, a decline in healthy eating habits has also been reported with an increase in snack and sugary drink consumption during the COVID-19 lockdown in children and adolescents. Whether and to what extent behavioral changes in activity, sleep, and eating habits impact children and adolescents into adulthood is unknown and should be a cause of concern and a top priority for research.

Obesity in adults is strongly associated with many serious medical complications that impair quality of life and lead to increased morbidity and mortality. In recent years, strong evidence has mounted to show that childhood and adolescent obesity is also a major medical and public health problem. Childhood and adolescent obesity is strongly associated with cardiac hypertrophy, increased blood pressure, increased cardiac epicardial adipose tissue, diastolic and systolic dysfunction, increased carotid intima-media thickness (cIMT), increased arterial stiffness, endothelial dysfunction, and impaired cardiac autonomic function [8-10]. Moreover, obesity in children and adolescents is associated with impaired glucose tolerance, insulin resistance, type 2 diabetes mellitus (T2DM), and dyslipidemia, among many other physiological and psychological derangements [10].

Childhood and adolescent obesity affects subjects at early ages and translates into the increased prevalence of cardiovascular risk factors and cardiovascular disease in adulthood. Four large studies of cardiovascular risk factors that initially involved children followed the participants into adulthood: the Bogalusa Heart Study (conducted in the United States), the Muscatine Study (United States), the Childhood Determinants of Adult Health (CDAH) study (Australia), and the Cardiovascular Risk in Young Finns Study (Finland). An analysis of four large cohorts, 
including more than 6000 children (mean age, 11 years) followed up for more than 23 years into adulthood, showed a positive correlation between childhood BMI and the following cardiovascular risk factors: hypertension, T2DM, LDL cholesterol, triglycerides, and cIMT [11]. Furthermore, the childhood and adolescent obesity-associated increase in cardiovascular risk factors in adulthood is also associated with increased cardiovascular events. A longterm follow-up study of more than a quarter of a million Danish children (age range, 7-13 years) into adulthood for more than 45 years showed that higher BMI during childhood is associated with an increased risk of fatal and nonfatal coronary heart disease events in adulthood [12]. These findings were validated in another large study of 2.3 million Israeli adolescents (mean age, 17 years) followed into adulthood. Adolescent obesity was associated with increased cardiovascular and all-cause mortality in adulthood [13]. In summary, childhood and adolescent obesity is a medical and public health concern that requires immediate action to prevent its deleterious effects into adulthood.

The most common obesity diagnostic tool in the clinic is the BMI, calculated as weight in kilograms divided by height squared in meters. BMI is an attractive parameter for diagnosing obesity in the clinical setting due to its simplicity. However, BMI has a series of severe limitations. It does not distinguish between fat and lean mass, does not account for fat distribution pattern, and is not adjusted by

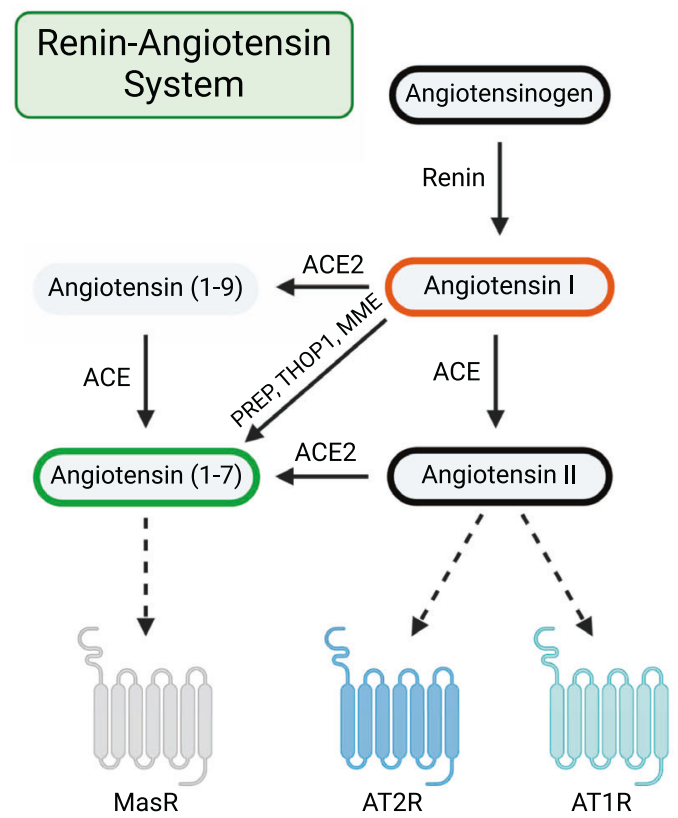

Fig. 1 Crosstalk between the renin-angiotensin system (RAS) and the kallikrein-kinin system (KKS). Peptides regulated by obesity in adolescents as reported by Fernandes et al. [17] are shown with bolded borders. Upregulated peptides are shown in red, downregulated peptides are shown in green, and nonregulated peptides are shown in black. Nomenclature key: abbreviations used: gene name (gene symbol). ACE angiotensin-converting enzyme, ACE2 angiotensin- age or ethnicity. Moreover, the novel and highly controversial concept of metabolically healthy obesity raises further concerns regarding the generalized validity of BMI for obesity diagnosis. Metabolically healthy OB subjects are individuals who are classified as OB based on BMI but present an apparently healthy phenotype [14, 15]. For all the aforementioned concerns, there is an active pursuit in the search for novel obesity biomarkers that could bring light to the diagnosis and treatment of this devastating disease [16].

In the current issue of Hypertension Research, Fernandes et al. [17] analyzed circulating levels of the renin-angiotensin system (RAS) and the kallikrein-kinin system (KKS) as potential obesity biomarkers in adolescence.

The RAS, a major regulator of blood pressure and metabolism, is divided into classical (vasoconstrictorprofibrotic-proinflammatory) and nonclassical (vasodilatorantifibrotic-anti-inflammatory) arms, producing opposite physiological effects $[18,19]$. In addition to the systemic RAS, the local RAS in organs, such as the brain, heart, pancreas, and adipose tissue, has been described [20]. In the classical RAS arm, angiotensinogen (AGT) is cleaved by renin to generate angiotensin I (Ang I), which is then converted to angiotensin II (Ang II) by angiotensin-converting enzyme (ACE) (Fig. 1). Ang II binds and activates the Ang II type 1 receptor, causing vasoconstriction and sodium retention, which increases blood pressure. Ang II also binds and activates Ang II type 2 receptor, causing vasodilation and decreasing inflammation, fibrosis, and

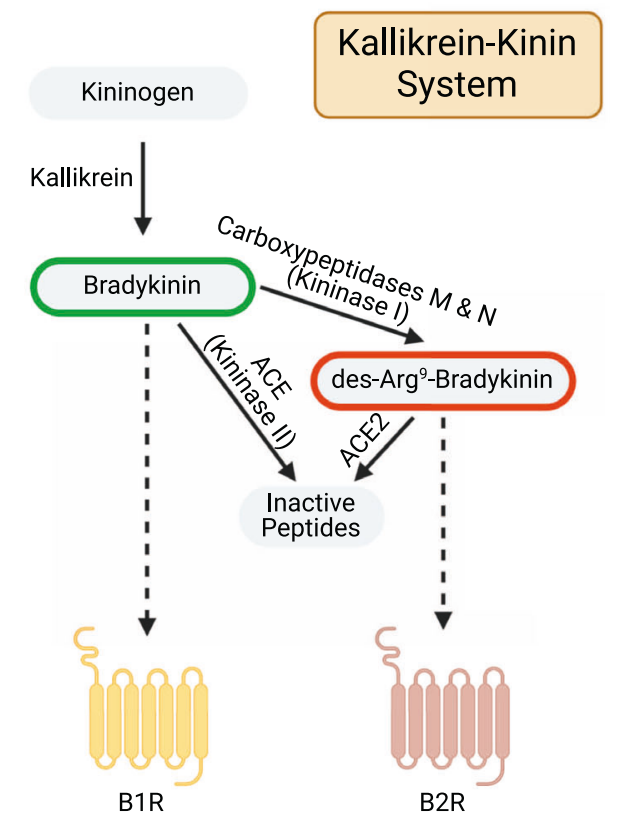

converting enzyme 2, AT1R angiotensin II type 1 receptor (AGTR1), AT2R angiotensin II type 2 receptor (AGTR2), MasR Mas receptor (Mas1), MME membrane metallo-endopeptidase (also known as neprilysin), PREP prolyl endopeptidase, THOP1 thimet oligopeptidase 1, carboxypeptidase M (CPM), carboxypeptidase N (CPN1, CPN2), B1R bradykinin receptor B1 (BDKRB1), B2R bradykinin receptor B2 (BDKRB2) 
hypertrophy. In the nonclassical RAS arm, angiotensinconverting enzyme 2 (ACE2) converts Ang II to angiotensin (1-7) (Ang (1-7)), which binds to the Mas receptor, opposing most of the physiological actions of Ang II and causing vasodilation and natriuresis. Ang (1-7) can also be generated by other alternative pathways by either sequential proteolysis of Ang I by ACE2 and ACE or by the action of either membrane metallo-endopeptidase (also known as neprilysin), prolyl endopeptidase, or thimet oligopeptidase 1 on Ang (1-7).

In the KKS, kininogen is cleaved by kallikrein to bradykinin, which can then be converted to des-Arg ${ }^{9}$-bradykinin by carboxypeptidases $\mathrm{M}$ and $\mathrm{N}$ (also known as kininase II). Bradykinin and des-Arg ${ }^{9}$-bradykinin bind preferentially to bradykinin receptors $\mathrm{B} 2$ and $\mathrm{B} 1$, respectively. Bradykinin and des- $\mathrm{Arg}^{9}$-bradykinin are converted to inactive peptides by ACE (also known as kininase II) and $\mathrm{ACE} 2$, respectively. Bradykinin and des-Arg ${ }^{9}$-bradykinin are implicated in a wide range of biological phenomena, including pain, inflammation, vasodilation, increased vascular permeability, and natriuresis.

In the current issue of Hypertension Research, Fernandes et al. [17] reported an exhaustive analysis of the circulating RAS and KKS peptides in healthy adolescents to identify obesity biomarkers. Healthy adolescent volunteers (11-17 years old) were classified into normal weight, $\mathrm{OW}, \mathrm{OB}$, and morbidly obese (MO) subjects based on their BMI. Circulating levels of Ang I, Ang II, Ang (1-7), bradykinin, and des-Arg ${ }^{9}$-bradykinin were quantified by high-performance liquid chromatography. Moreover, circulating levels of AGT, renin, and ACE activity were quantified. Furthermore, an impressive set of anthropometric and clinical chemistry parameters, metabolic determinations, and blood pressure was obtained from all subjects, allowing multiple comparisons and correlations that will surely open the door to new studies in the future. Analysis of the circulating RAS showed that Ang I was positively and Ang (1-7) was negatively correlated with BMI. On the other hand, analysis of the circulating KKS showed that bradykinin was negatively and des-Arg'-bradykinin was positively correlated with BMI. ACE2 is involved in Ang (1-7) synthesis and des-Arg'-bradykinin degradation (Fig. 1). Ang (1-7)negative and des-Arg ${ }^{9}$-bradykinin-positive correlations with BMI strongly suggest decreased ACE2 activity in obesity. ACE2 is ubiquitously expressed in kidney, heart, lung, adipose, liver, skeletal muscle, pancreas, gut, and other tissues [21]. Although it is not possible to know with certainty the tissue(s) responsible for the decreased ACE2 activity in obesity in this study, it is possible to speculate that adipose-tissue ACE2 could be partially responsible for such systemic effects. Supportive of the aforementioned speculation is the novel finding that ACE2 mRNA expression in subcutaneous adipose tissue negatively correlates with BMI [22]. However, other studies have shown that weight loss in OW and OB individuals causes a decrease in ACE2 mRNA expression in subcutaneous adipose tissue $[23,24]$. Whether those observed changes in ACE2 mRNA expression translate into changes in the levels or activity of ACE2 protein is unknown. Determination of ACE2 expression and/or activity in adipose tissue in cohort studies by Fernandes and colleagues or a similar study will settle the question of the tissue(s) involved in decreased systemic levels of the ACE2 product Ang (1-7) and the ACE2 substrate des-Arg ${ }^{9}$-bradykinin.

Fernandes et al. [17] reported an increase in both systolic and diastolic blood pressure in otherwise healthy, MO adolescents [17]. Moreover, the researchers found a positive correlation between systolic blood pressure (SBP) and circulating levels of Ang I and des-Arg ${ }^{9}$-bradykinin. Furthermore, they also observed a negative correlation between SBP and circulating levels of Ang (1-7) and bradykinin. These exciting human clinical findings nicely complement preclinical studies of diet-induced obesity in mice. A highfat diet decreased plasma Ang (1-7) in male mice but increased plasma Ang (1-7) in female mice [25]. The increase in circulating Ang (1-7) in female mice fed a highfat diet was correlated with an increase in adipose-tissue ACE2 activity; however, no changes were observed in ACE2 activity in male mice. Moreover, the decrease in plasma Ang (1-7) in male mice fed a high-fat diet was associated with an increase in SBP. However, there were no significant changes in SBP in female mice fed a low- or high-fat diet. In view of those sex-difference findings in rodents, it would have been intriguing to have included sex as an additional variable in the study by Fernandes and colleagues, although a larger cohort would have probably been needed to have enough statistical power. We expect that such studies could be performed in the future to shed light on ACE2 sex-specific regulation.

In summary, the report by Fernandes et al. [17] is an interesting clinical study that will surely open the field to future studies. Some exciting questions that remain to be answered, among many others, include the following: (1) Would the current findings be affected when the same study is performed in a population including subjects with other comorbidities? (2) Are the current findings sex dependent? (3) Does physical activity and/or a healthy lifestyle intervention revert the obesity-induced changes in the RAS and KKS? In conclusion, this study shows that circulating RAS and KKS peptides are potential obesity biomarkers that could help identify adolescents at higher risk of obesityrelated complications. We applaud the initiative of Fernandes and colleagues to tackle this major medical and public health problem. Our children and adolescents deserve a healthier life. The time to act is now. 
Funding This work was supported by National Institutes of Health National Institute of General Medical Sciences grant P20GM121334 (LLYC and DGR) and National Institute of Diabetes and Digestive and Kidney Diseases grant R21DK113500 (DGR). The content is solely the responsibility of the authors and does not necessarily represent the official views of the National Institutes of Health.

\section{Compliance with ethical standards}

Conflict of interest The authors declare no competing interests.

Publisher's note Springer Nature remains neutral with regard to jurisdictional claims in published maps and institutional affiliations.

\section{References}

1. United Nations Children's Fund (UNICEF), WHO, International Bank for Reconstruction, Bank DTW. Levels and trends in child malnutrition: key findings of the 2020, Edition of the Joint Child Malnutrition Estimates. Geneva: World Health Organization; 2020.

2. Templin T, Cravo Oliveira Hashiguchi T, Thomson B, Dieleman $\mathrm{J}$, Bendavid $\mathrm{E}$. The overweight and obesity transition from the wealthy to the poor in low- and middle-income countries: A survey of household data from 103 countries. PLoS Med. 2019;16:e1002968.

3. Hales CM, Carroll MD, Fryar CD, Ogden CL. Prevalence of obesity among adults and youth: United States, 2015-2016. NCHS data brief, No. 288. Hyattsville, MD: National Center for Health Statistics; 2017.

4. Pietrobelli A, Pecoraro L, Ferruzzi A, Heo M, Faith M, Zoller T, et al. Effects of COVID-19 lockdown on lifestyle behaviors in children with obesity living in Verona, Italy: a longitudinal study. Obesity. 2020;28:1382-5.

5. Yang S, Guo B, Ao L, Yang C, Zhang L, Zhou J, et al. Obesity and activity patterns before and during COVID-19 lockdown among youths in China. Clin Obes. 2020;10:e12416.

6. Moore SA, Faulkner G, Rhodes RE, Brussoni M, Chulak-Bozzer $\mathrm{T}$, Ferguson LJ, et al. Impact of the COVID-19 virus outbreak on movement and play behaviours of Canadian children and youth: a national survey. Int J Behav Nutr Phys Act. 2020;17:85.

7. Dunton GF, Do B, Wang SD. Early effects of the COVID-19 pandemic on physical activity and sedentary behavior in children living in the U.S. BMC Public Health. 2020;20:1351.

8. Cote AT, Harris KC, Panagiotopoulos C, Sandor GG, Devlin AM. Childhood obesity and cardiovascular dysfunction. J Am Coll Cardiol. 2013;62:1309-19.

9. Bacha F, Gidding SS. Cardiac abnormalities in youth with obesity and type 2 diabetes. Curr Diab Rep. 2016;16:62.

10. May AL, Kuklina EV, Yoon PW. Prevalence of cardiovascular disease risk factors among US adolescents, 1999-2008. Pediatrics. 2012;129:1035-41.
11. Juonala M, Magnussen CG, Berenson GS, Venn A, Burns TL, Sabin MA, et al. Childhood adiposity, adult adiposity, and cardiovascular risk factors. N Engl J Med. 2011;365:1876-85.

12. Baker JL, Olsen LW, Sorensen TI. Childhood body-mass index and the risk of coronary heart disease in adulthood. N Engl J Med. 2007;357:2329-37.

13. Twig G, Yaniv G, Levine H, Leiba A, Goldberger N, Derazne E, et al. Body-mass index in 2.3 million adolescents and cardiovascular death in adulthood. N Engl J Med. 2016;374:2430-40.

14. Bluher M. Metabolically healthy obesity. Endocr Rev. 2020; 41:405-20.

15. Iacobini C, Pugliese G, Blasetti Fantauzzi C, Federici M, Menini S. Metabolically healthy versus metabolically unhealthy obesity. Metabolism. 2019;92:51-60.

16. Nimptsch K, Konigorski S, Pischon T. Diagnosis of obesity and use of obesity biomarkers in science and clinical medicine. Metabolism. 2019;92:61-70.

17. Fernandes FB, Fernandes AB, Febba ACS, Leite APO, Leite CA, Vitalle MSS, et al. Association of Ang-(1-7) and des-Arg(9)BK as new biomarkers of obesity and cardiometabolic risk factors in adolescents. Hypertens Res. 2021. https://doi.org/10.1038/ s41440-021-00618-0.

18. Santos RAS, Oudit GY, Verano-Braga T, Canta G, Steckelings UM, Bader M. The renin-angiotensin system: going beyond the classical paradigms. Am J Physiol Heart Circ Physiol. 2019;316: H958-70.

19. Li XC, Zhang J, Zhuo JL. The vasoprotective axes of the reninangiotensin system: physiological relevance and therapeutic implications in cardiovascular, hypertensive and kidney diseases. Pharm Res. 2017;125:21-38.

20. Paul M, Poyan Mehr A, Kreutz R. Physiology of local reninangiotensin systems. Physiol Rev. 2006;86:747-803.

21. Gembardt F, Sterner-Kock A, Imboden H, Spalteholz M, Reibitz F, Schultheiss HP, et al. Organ-specific distribution of ACE2 mRNA and correlating peptidase activity in rodents. Peptides. 2005;26:1270-7.

22. El-Sayed Moustafa JS, Jackson AU, Brotman SM, Guan L, Villicana S, Roberts AL, et al. ACE2 expression in adipose tissue is associated with COVID-19 cardio-metabolic risk factors and cell type composition. medRxiv. 2020. https://doi.org/10.1101/2020. 08.11.20171108.

23. Al Heialy S, Hachim MY, Senok A, Gaudet M, Abou Tayoun A, Hamoudi R, et al. Regulation of angiotensin-converting enzyme 2 in obesity: implications for COVID-19. Front Physiol. 2020;11:555039.

24. Li L, Spranger L, Soll D, Beer F, Brachs M, Spranger J, et al. Metabolic impact of weight loss induced reduction of adipose ACE-2 - potential implication in COVID-19 infections? Metabolism. 2020;113:154401.

25. Gupte M, Thatcher SE, Boustany-Kari CM, Shoemaker R, Yiannikouris F, Zhang X, et al. Angiotensin converting enzyme 2 contributes to sex differences in the development of obesity hypertension in C57BL/6 mice. Arterioscler Thromb Vasc Biol. 2012;32:1392-9. 